\title{
Repositorios y redes sociales académicas, dos vehículos para un mismo conocimiento científico
}

Alexandre López-Borrull | Estudis de ciències de la información i la comunicació, Universitat Oberta de Catalunya

URL de la contribución <www.iaph.es/revistaph/index.php/revistaph/article/view/4660>

El presente debate sobre repositorios y redes sociales académicas es ciertamente interesante y puede apuntar a múltiples visiones complementarias tal como estamos viendo por las aportaciones, distintas y valiosas, que se están publicando en el debate creado y coordinado por nuestra muy experta y muy activista Reme Melero.

Teniendo la oportunidad de poder opinar sobre cómo deberían colaborar los dos tipos de productos, quisiera hacerlo desde el punto de vista de un profesional de la información pero, sobre todo, de un científico que tiene una actitud y unos valores, relacionados con el acceso y la ciencia abierta, y que además convive con una presión del sistema y su propia Universidad por el nuevo citius, altius fortius académico que sería el "publica más, sé más citado y ten más impacto". Mi aportación, además, está escrita en el período de transición entre la ciencia a. c. (antes del coronavirus) y aquella nueva ciencia, la d. c., que puede venir, más abierta, seguro, pero también con un papel más central del conocimiento científico a nivel social y sobre todo político. Quisiera pues, hacer la siguientes consideraciones:

\section{No eres tú, es la visibilidad}

A menudo, entre científicos emerge un debate sobre cuál debe ser la forma más adecuada para difundir más y mejor en abierto, incluso entre aquellos que tienen claras las ventajas. En dicho debate, parece que se intenten contraponer las dos estrategias, como si los científicos tuvieran que tomar partido entre repositorios o redes sociales. Como investigador, lo primero que debo considerar es el hecho de que a veces parece un encrucijada elegir entre el repositorio y la red social académica, cuando finalmente acaban siendo dos momentos de un mismo proceso continuo, la difusión del conocimiento

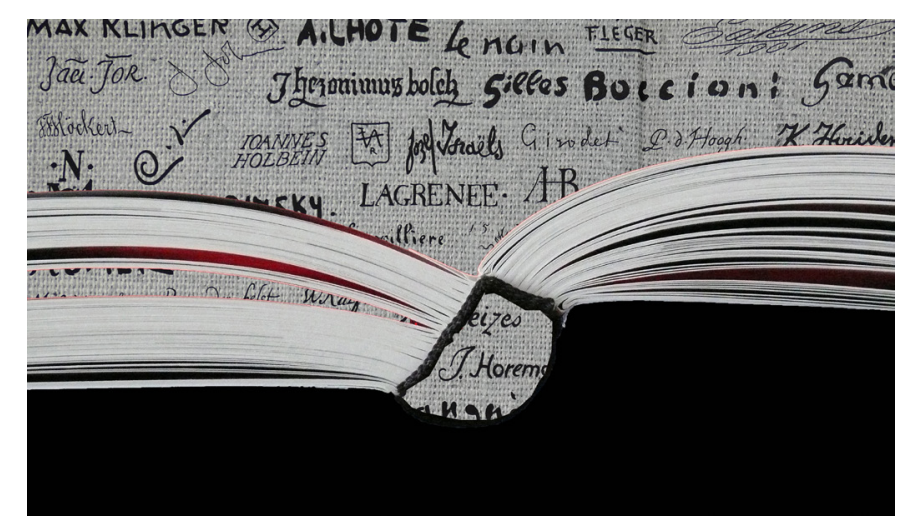

La visibilidad es la fuerza motriz para el depósito en abierto

generado en tu investigación y publicado en forma de documento (OLLÉ; LÓPEZ-BORRULL, 2018).

De alguna forma, debemos considerar que la visibilidad pasa a ser la fuerza motriz para el depósito en abierto. Como describieron Beel y sus colaboradores (2010), estamos hablando de que los científicos deben conocer mejor las técnicas y herramientas relacionadas no sólo con el Search Engine Optimization (SEO), sino sobre el Academic SEO (ASEO), por cuanto se refiere a las estrategias para ser localizado a través de los buscadores.

Es, pues, en los buscadores donde debemos entender a menudo las reglas de juego. Últimamente, parece que las redes sociales académicas como Academia y ResearchGate (sobre todo) tienen una posición de prioridad en las búsquedas en Google. Por los enlaces y el PageRank que utiliza Google, la búsqueda de artículos muestran primero los resultados en ResearchGate, según mi experiencia. Por tanto, parece lógico que la difusión de artículos (preprints y publicados) se haga también en este tipo de redes sociales, siempre consi- 
a debate Repositorios y redes sociales académicas para la transferencia del conocimiento abierto

| coordina Remedios Melero Melero

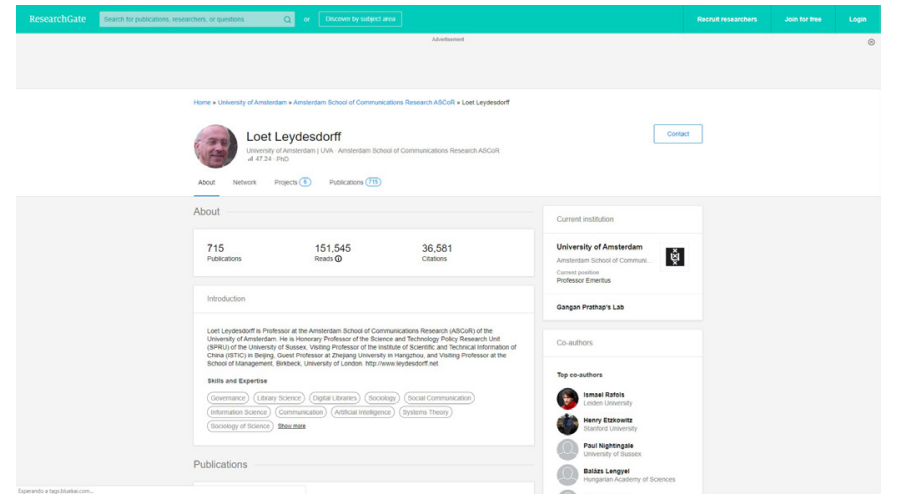

Perfil del científico Loet Leydesdorff en ResearchGate

derando la posibilidad legal de llevarlo a cabo, aunque sobre este aspecto volveremos más tarde. Pero, ¿solo interesa la visibilidad en los primeros resultados de la búsqueda en Google? Creo que no, por cuanto los científicos también quieren que su producción (y sus citas) también aparezcan en su perfil de Google Scholar Citations, y para ello los repositorios institucionales tienen un papel importante.

A menudo me recuerda la visión entre el consumo de redes sociales y de medios de comunicación que están entrecruzados, siendo precisamente las redes sociales donde la gente más se informa de los contenidos de los medios de comunicación (COMISIÓN EUROPEA, 2018), de forma que es una falsa dicotomía.

\section{¿Es posible acompasar distintos modelos de negocio $y$ retos de futuro?}

Parece evidente que los repositorios y las redes sociales académicas parten de unas dinámicas y unos objetivos diferentes. Sin duda, la existencia de un modelo de negocio en el caso de las redes sociales académicas es un factor a considerar, sobre todo en un futuro incierto. Así como que el resto de social media basan su engagement en la experiencia enriquecedora para el usuario, el atractivo y el prestigio que puedan aportar y, sobre todo, la continua presencia en la red, formando un pez que se muerde la cola, todo ello acompañado de un envío masivo de correos electrónicos en función de las interacciones que se haya prefijado.

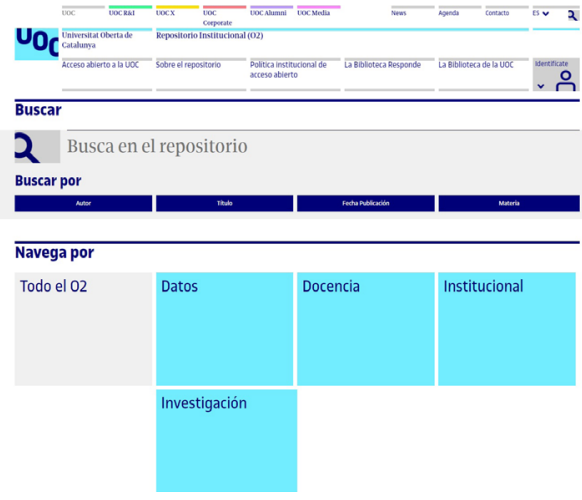

Interfaz de búsqueda del repositorio institucional de la UOC

Es por muchos de estos aspectos que las redes sociales académicas deben demostrar la sostenibilidad y la monetización a futuro, con perfiles premium o de la forma que sea. ¿Puede Academia.edu, por poner un ejemplo, ser el MySpace del mundo académico, del cual ya nadie se acuerda? Sin duda, y es por ello que las redes sociales deben apostar por un valor añadido propio y diferencial. La ciencia abierta debería ser uno de los motivos, nadie como ellos puede poner en relación científicos de múltiples disciplinas y países, y de hecho con los proyectos y los debates intentan emular ágoras académicas. En cambio, los repositorios deben seguir acompasando las estrategias de las instituciones donde residen y ayudar al objetivo, a veces denostado pero básico en un entorno demasiado dinámico, de la preservación a futuro.

A su vez, parece lógico que los derechos y deberes de repositorios y redes sociales académicas converjan. No podía ser que sólo unos tuvieran la responsabilidad de controlar la propiedad intelectual de todo aquello que se deposita en ellos (ACADEMIC, 2018). Las exigencias de las grandes editoriales con ResearchGate (RESEARCHGATE, 2018) por ejemplo han servido también para romper con una cierta idea inicial de que aquello podía ser como un Sci-hub alimentado por los propios científicos, un sistema P2P como lo fue Napster. Con los controles actuales en el momento de colgar archivos (básicamente un intento de disclaimer para los repositorios) se puede estar reconduciendo el asunto. 


\section{Privacidad versus ciencia abierta}

En relación a otro de los apuntes que quería hacer, el repositorio institucional describe el global de nuestra institución, mientra que los perfiles de los investigadores en las redes sociales académicas los describen a ellos. En las redes sociales generalistas, principalmente Facebook, hemos visto cómo uno de los principales problemas de futuro que plantea (el otro es la desinformación que campa a sus anchas en dichos entornos) es la privacidad. Privacidad de nuestros datos personales. Sí, el intercambio de datos gratuitamente parece estar descompensándose por los usos y las perversiones en su comportamiento. Parece curioso que en el caso de las redes sociales académicas las quejas no han provenido de los autores sino de las editoriales. Entre todos y todas estamos alimentando un depósito que puede plantearse, como las grandes editoriales, pensar en posibles proyectos de minería de datos científicos (VILLATORO, 2019), alimentados por nosotros.

\section{Por último, algo en común, contenidos de valor en un contexto de desinformación}

No quisiera dejar de poner en valor el papel (y la responsabilidad) que las redes sociales académicas y los repositorios tienen no sólo para difundir conocimiento científico, sino para llevarlo a cabo en épocas de desinformación científica. En efecto, nunca como en esta crisis de salud pública habíamos podido comprobar cómo las fake news y fake science tienen presencia en todas las redes sociales generalistas (principalmente Whatsapp, Facebook y Youtube). Este campo es una preocupación para las propias redes sociales (una de sus principales amenazas de futuro), que deberían tomar cartas para dibujar escenarios de validación y curación de contenidos, labor en la cual los repositorios siempre estarán uno o dos pasos por delante, porque ya tienen de su lado los servicios bibliotecarios universitarios.

Como conclusión quisiera haber aportado nuevas visiones sobre un debate que para el científico no se cerrará en breve. La necesidad acuciante entre acreditar la producción e incrementar su impacto hace que en las estrategias de difusión debas acompasar todas las herramientas al alcance. Los repositorios institucionales tienen un papel central, aunque también los tienen los disciplinares, que hemos dejado fuera en nuestra aportación. La difusión de preprints y las estructuras flexibles y preparadas para la difusión de los datos de investigación deben marcar la visión en un futuro, con revistas y plataformas más líquidas y con indicadores y métricas por doquier. Podrá ser más lento de lo deseable, pero no tiene vuelta atrás.

\section{BIBLIOGRAFÍA}

- ACADEMIC Publishers Form a Coalition with ResearchGate. ResearchGate vs. Publishers. Enago academy. 8 de mayo de $2018<$ https://www.enago.com/academy/academic-publishersform-a-coalition-with-researchgate/> [Consulta: 05/05/2020]

- COMISIÓN EUROPEA (2018) Fake news and disinformation online. Flash Eurobarometer 464 <http://ec.europa.eu/ commfrontoffice/publicopinion/index.cfm/ResultDoc/download/ DocumentKy/82797> [Consulta: 05/05/2020]

- BEEL, J.; GIPP, B.; WILDE, E. (2010) Academic Search Engine Optimization (ASEO): Optimizing Scholarly Literature for Google Scholar and Co. Journal of Scholarly Publishing, vol. $41,{ }^{\circ}{ }^{2}$ 2, pp. 176-190 <https://utpjournals.press/doi/10.3138/ jsp.41.2.176> [Consulta: 05/05/2020]

- OLLÉ, C.; LÓPEZ-BORRULL, A. (2017) Redes sociales y altmetrics: nuevos retos para las revistas científicas. En ABADAL, E. (ed.) Revistas científicas. Situación actual y retos de futuro. Barcelona: Edicions Universitat de Barcelona, ISBN 978-84-9168-004-8 <http://eprints.rclis.org/32138/> [Consulta: 05/05/2020]

- RESEARCHGATE Removes 1.7 Million Articles In Copyright Conflict. ResearchGate vs. Publishers. Enago academy, 8 de mayo de 2018 <https://www.enago.com/academy/ researchgate-removes-1-7-million-articles-copyright-conflict/> [Consulta: 05/05/2020]

- VILLATORO, F. R. (2019) El descubrimiento científico usando minería de artículos científicos. Blog La ciencia de la mula Francis, 4 de julio de $2019<$ https://francis.naukas. com/2019/07/04/el-descubrimiento-cientifico-usando-mineriade-articulos-cientificos/> [Consulta: 05/05/2020] 